RIMS-985

YITP/K-1076

YITP/U-94-22

SULDP-1994-4

June 1994

\title{
Subalgebras of $W_{1+\infty}$ and Their Quasifinite Representations
}

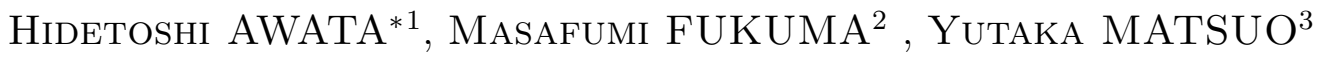 \\ and SATORU ODAKE' \\ ${ }^{1}$ Research Institute for Mathematical Sciences \\ Kyoto University, Kyoto 606, Japan \\ ${ }^{2}$ Yukawa Institute for Theoretical Physics \\ Kyoto University, Kyoto 606, Japan \\ ${ }^{3}$ Uji Research Center, Yukawa Institute for Theoretical Physics \\ Kyoto University, Uji 611, Japan \\ ${ }^{4}$ Department of Physics, Faculty of Liberal Arts \\ Shinshu University, Matsumoto 390, Japan
}

\begin{abstract}
We propose a series of new subalgebras of the $W_{1+\infty}$ algebra parametrized by polynomials $p(w)$, and study their quasifinite representations. We also investigate the relation between such subalgebras and the $\hat{\mathrm{gl}}(\infty)$ algebra. As an example, we investigate the $W_{\infty}$ algebra which corresponds to the case $p(w)=w$, presenting its free field realizations and Kac determinants at lower levels.
\end{abstract}

\section{hep-th/9406111}

\footnotetext{
*JSPS fellow

1 e-mail address : awata@kurims.kyoto-u.ac.jp

2 e-mail address : fukuma@yukawa.kyoto-u.ac.jp

3 e-mail address : yutaka@yukawa.kyoto-u.ac.jp

${ }^{4}$ e-mail address : odake@jpnyitp.yukawa.kyoto-u.ac.jp
} 


\section{Introduction}

It is known that there are several types of $W$ infinity algebras, including the $w_{\infty}$ algebra as the algebra of area preserving diffeomorphism of two-dimensional cylinder [1], the $W_{\infty}$ algebra as its deformation [2], and the $W_{1+\infty}$ algebra by adding a spin-1 current to $W_{\infty}$ [3]. The $W_{1+\infty}$ algebra may be regarded as the most fundamental because all other $W$ infinity algebras are obtained as its subalgebras [甘].

The representation theories of these algebras had not been developed after the early works [5, 6], because of the difficulty that there might exist infinitely many states at each energy level reflecting the infinite number of currents. The requirement that there exist only a finite number of states at each energy level is quite natural from the view point of the free-field realization, since such quasifiniteness condition is actually ensured when states are generated by (a finite number of) free-field oscillators. Recently Kac and Radul gave an elegant framework to set up the quasifinite condition for the $W_{1+\infty}$ algebra, and studied such representations in detail [7]. On the basis of their analysis, further studies were made for the $W_{1+\infty}$ algebra [8, 9, 10, 11] and the super $W_{1+\infty}$ algebra [12.

In this letter, we propose a systematic method to construct a family of subalgebras of the $W_{1+\infty}$ algebra, and then study their quasifinite representations. In particular, we investigate the $W_{\infty}$ algebra as a special case, and present the free field realizations and the Kac determinant formula at lower levels.

\section{Subalgebras of $W_{1+\infty}$}

The $W_{1+\infty}$ algebra is defined as a one-dimensional central extension of the Lie algebra of differential operators on the circle whose classical generators are $z^{n} D^{k}\left(D \equiv z \frac{d}{d z}, n \in \mathbb{Z}\right.$, $\left.k \in \mathbb{Z}_{\geq 0}\right)$. We denote the corresponding generators in the $W_{1+\infty}$ algebra by $W\left(z^{n} D^{k}\right)$ and the central charge by $C$. The commutation relations are defined by [0]

$$
\begin{aligned}
& {\left[W\left(z^{n} f(D)\right), W\left(z^{m} g(D)\right)\right] } \\
= & W\left(z^{n+m} f(D+m) g(D)\right)-W\left(z^{n+m} f(D) g(D+n)\right) \\
& +C \delta_{n+m, 0}\left(\theta(n \geq 1) \sum_{j=1}^{n} f(-j) g(n-j)-\theta(m \geq 1) \sum_{j=1}^{m} f(m-j) g(-j)\right),
\end{aligned}
$$

where $\theta(P)=1$ (or 0 ) when the proposition $P$ is true (or false).

In the above expression, $f(D)$ and $g(D)$ are arbitrary polynomials. However, the commutation relations still close even for a class of the polynomials which can be divided by a polynomial $p(D)$. In fact, if we set

$$
\widetilde{W}\left(z^{n} D^{k}\right)=W\left(z^{n} D^{k} p(D)\right)
$$


then their commutation relations will be written as

$$
\begin{aligned}
& {\left[\widetilde{W}\left(z^{n} f(D)\right), \widetilde{W}\left(z^{m} g(D)\right)\right] } \\
= & \widetilde{W}\left(z^{n+m} p(D+m) f(D+m) g(D)\right)-\widetilde{W}\left(z^{n+m} p(D+n) f(D) g(D+n)\right) \\
+C \delta_{n+m, 0}\left(\theta(n \geq 1) \sum_{j=1}^{n} p(-j) p(n-j) f(-j) g(n-j)\right. & \\
& \left.-\theta(m \geq 1) \sum_{j=1}^{m} p(-j) p(m-j) f(m-j) g(-j)\right) .
\end{aligned}
$$

We call the obtained subalgebra $W_{1+\infty}[p(D)]$. The motivation for our construction of such subalgebras is as follows. In the $W_{\infty}$ algebra, there is no spin-1 current. Since the current is expressed as $W\left(z^{n}\right)$ in $W_{1+\infty}$, the $W_{\infty}$ algebra will be obtained from $W_{1+\infty}$ simply by omitting this current. However, it is equivalent to taking $p(D)=D$ in the above expression.

By introducing $z^{n} e^{x D}$ as a generating series for $z^{n} D^{k}$, the above commutation relation can be rewritten in a simpler form:

$$
\begin{aligned}
{\left[\widetilde{W}\left(z^{n} e^{x D}\right), \widetilde{W}\left(z^{m} e^{y D}\right)\right]=} & \left(p\left(\frac{d}{d x}\right) e^{m x}-p\left(\frac{d}{d y}\right) e^{n y}\right) \widetilde{W}\left(z^{n+m} e^{(x+y) D}\right) \\
& -C p\left(\frac{d}{d x}\right) p\left(\frac{d}{d y}\right) \frac{e^{m x}-e^{n y}}{e^{x+y}-1} \delta_{n+m, 0}
\end{aligned}
$$

The basis of $W_{1+\infty}$ given in [3], $V_{n}^{i}=W_{n}^{i+2}$, is expressed as $W_{n}^{k+1}=W\left(z^{n} f_{n}^{k}(D)\right)$ $(k \geq 0)$,

$$
f_{n}^{k}(D)=\left(\begin{array}{c}
2 k \\
k
\end{array}\right)^{-1} \sum_{j=0}^{k}(-1)^{j}\left(\begin{array}{c}
k \\
j
\end{array}\right)^{2}[-D-n-1]_{k-j}[D]_{j}
$$

where $[x]_{n}=\prod_{j=0}^{n-1}(x-j)$ and $\left(\begin{array}{l}x \\ n\end{array}\right)=[x]_{n} / n$ !. On the other hand, the basis of $W_{\infty}$ given in [2], $\widetilde{V}_{n}^{i}=\widetilde{W}_{n}^{i+2}$, is now expressed as $\widetilde{W}_{n}^{k+2}=\widetilde{W}\left(z^{n} \tilde{f}_{n}^{k}(D)\right)(k \geq 0)$,

$$
\tilde{f}_{n}^{k}(D)=-\left(\begin{array}{c}
2(k+1) \\
k+1
\end{array}\right)^{-1} \sum_{j=0}^{k}(-1)^{j}\left(\begin{array}{c}
k \\
j
\end{array}\right)\left(\begin{array}{c}
k+2 \\
j+1
\end{array}\right)[-D-n-1]_{k-j}[D-1]_{j} .
$$

We remark that the Virasoro generators exist only if $\operatorname{deg} p(w) \leq 1$. In the case of $W_{\infty}$, the Virasoro generator $L_{n}$ is given by $L_{n}=-\widetilde{W}\left(z^{n}\right)$ whose central charge, $\tilde{c}_{V i r}$, is related to $C$ as $\tilde{c}_{V i r}=-2 C$ [4]. For $\operatorname{deg} p(w) \geq 2$, we extend the algebra introducing the $L_{0}$ operator such as to count the energy level, $\left[L_{0}, \widetilde{W}\left(z^{n} f(D)\right)\right]=-n \widetilde{W}\left(z^{n} f(D)\right)$.

\section{Quasifinite Representations}

We consider the irreducible quasifinite highest weight representations of $W_{1+\infty}[p(D)]$, following [0]. 
The highest weight state $|\lambda\rangle$ is characterized by

$$
\begin{aligned}
\widetilde{W}\left(z^{n} D^{k}\right)|\lambda\rangle & =0 & & (n \geq 1, k \geq 0), \\
\widetilde{W}\left(D^{k}\right)|\lambda\rangle & =\widetilde{\Delta}_{k}|\lambda\rangle & & (k \geq 0) .
\end{aligned}
$$

It is convenient to introduce the generating function for the highest weights $\widetilde{\Delta}_{k}$,

$$
\widetilde{\Delta}(x)=-\sum_{k=0}^{\infty} \widetilde{\Delta}_{k} \frac{x^{k}}{k !},
$$

which is the eigenvalue of $-\widetilde{W}\left(e^{x D}\right) ; \widetilde{W}\left(e^{x D}\right)|\lambda\rangle=-\widetilde{\Delta}(x)|\lambda\rangle$. By definition, $\widetilde{\Delta}(x)$ must be regular at $x=0$. To study the representation of $W_{1+\infty}[p(D)]$, we first consider the representation of $W_{1+\infty}$ whose restriction agrees with the representation of $W_{1+\infty}[p(D)]$. Such a representation of $W_{1+\infty}$ always exists. The weight $\Delta(x)$, which is defined by $W\left(e^{x D}\right)|\lambda\rangle=-\Delta(x)|\lambda\rangle$, satisfies

$$
p\left(\frac{d}{d x}\right) \Delta(x)=\widetilde{\Delta}(x)
$$

The representation of the $W_{1+\infty}$ associated with that of $W_{1+\infty}[p(D)]$ is not uniquely determined, and we will fix one representative $\Delta(x)$.

The quasifinite representation of $W_{1+\infty}$ with $\Delta(x)$ is characterized by characteristic polynomials $b_{n}(w)(n=1,2,3, \cdots)$ [7] (see also [10]):

$$
b_{n}(w)=\operatorname{lcm}(b(w), b(w-1), \cdots, b(w-n+1)),
$$

where $b(w)=b_{1}(w)$ is the minimal-degree monic polynomial satisfying the differential equation,

$$
b\left(\frac{d}{d x}\right)\left(\left(e^{x}-1\right) \Delta(x)+C\right)=0 .
$$

Quasifinite condition is the requirement that there exist a finite number of states at each energy level. This means that $\widetilde{W}\left(z^{-n} f(D)\right)|\lambda\rangle$ is a null state for sufficiently highdegree polynomial $f(w)$. In other words, the set $\tilde{I}_{-n}=\left\{f(w) \in \mathbb{C}[w]\left|\widetilde{W}\left(z^{-n} f(D)\right)\right| \lambda\right\rangle=$ null state $\}$ contains an ideal of $\mathbb{C}[w]$ generated by some polynomial. We can show that if there exist a finite number of states at level 1 , then this is also the case at any level. Suppose that $\widetilde{W}\left(z^{-1} f(D)\right)|\lambda\rangle$ is a null state, then

$$
\begin{aligned}
0 & =\widetilde{W}\left(z e^{x(D+1)}\right) \widetilde{W}\left(z^{-1} f(D)\right)|\lambda\rangle \\
& =f\left(\frac{d}{d x}\right)\left(\left(p\left(\frac{d}{d x}\right) e^{x}-p\left(\frac{d}{d x}-1\right)\right) \widetilde{\Delta}(x)+C p(0) p(-1)\right)|\lambda\rangle \\
& =f\left(\frac{d}{d x}\right) p\left(\frac{d}{d x}\right) p\left(\frac{d}{d x}-1\right)\left(\left(e^{x}-1\right) \Delta(x)+C\right)|\lambda\rangle .
\end{aligned}
$$

Therefore, $\Delta(x)$ is the weight for the quasifinite representation. 
For $\operatorname{deg} p(w) \leq 2$, we can prove that $\tilde{I}_{-n}$ is an ideal of $\mathbb{C}[w]$, as is the case for $W_{1+\infty}$. However, this is no longer true when $\operatorname{deg} p(w) \geq 3$. In the following, we make an assumption that $\tilde{I}_{-n}$ is an ideal of $\mathbb{C}[w]$. Since $\mathbb{C}[w]$ is a principal ideal domain, $\tilde{I}_{-n}$ is generated by a monic polynomial $\tilde{b}_{n}(w)$. We call the $\tilde{b}_{n}(w)$ 's $(n=1,2,3, \ldots)$ the characteristic polynomials for the highest weight representation.

The characteristic polynomials $\tilde{b}_{n}(w)$ are related to each other as follows:?

(i) $\tilde{b}_{n}(w)$ divides both of $p(w+m) \tilde{b}_{n+m}(w+m)$ and $p(w-n-m) \tilde{b}_{n+m}(w){ }^{\forall} m \in \mathbb{Z} \geq 0$,

(ii) $p(w) p(w-n) \tilde{b}_{n}(w)$ is divided by $f_{n}(w)$.

The $\tilde{b}_{n}(w)$ 's are determined as minimal-degree polynomials satisfying (i) and (ii). Here $f_{n}(w)$ is the minimal-degree, monic polynomial satisfying the following differential equation:

$$
f_{n}\left(\frac{d}{d x}\right) \sum_{j=0}^{n-1} e^{j x}\left(\left(e^{x}-1\right) \Delta(x)+C\right)=0 .
$$

Note that $f_{1}(w)=b(w)$, and $b_{n}(w)$ is divided by $f_{n}(w)$. The property (i) is derived from the null state condition, $\widetilde{W}\left(z^{m} e^{x D}\right) \widetilde{W}\left(z^{-n-m} \tilde{b}_{n+m}(w)\right)|\lambda\rangle=$ null state. The property (ii) is derived from the following null state condition,

$$
\begin{aligned}
0 & =\widetilde{W}\left(z^{n} e^{x(D+n)}\right) \widetilde{W}\left(z^{-n} \tilde{b}(D)\right)|\lambda\rangle \\
& =\tilde{b}\left(\frac{d}{d x}\right) p\left(\frac{d}{d x}\right) p\left(\frac{d}{d x}-n\right) \sum_{j=0}^{n-1} e^{j x}\left(\left(e^{x}-1\right) \Delta(x)+C\right)|\lambda\rangle .
\end{aligned}
$$

In almost all cases, the relation $f_{n}(w)=b_{n}(w)$ holds. Then, we can prove that the characteristic polynomials $\tilde{b}_{n}(w)$ are given by

$$
\tilde{b}_{n}(w)=\frac{b_{n}(w)}{\operatorname{gcd}\left(b_{n}(w), p(w) p(w-n)\right)} .
$$

Although eq. (15) satisfies both (i) and (ii) in general, it might not be minimal-degree polynomials. Therefore, for such special cases, we must solve (i) and (ii) directly. In contrast to $W_{1+\infty}$, the $\tilde{b}_{n}(w)$ 's may not be determined by $\tilde{b}_{1}(w)$ (and $p(w)$ ) alone.

\section{Correspondence with $\hat{\mathrm{gl}}(\infty)$}

As was demonstrated in [7, 10], the quasifinite representation of $W_{\infty}$ can be alternatively expressed in terms of $\hat{\mathrm{gl}}(\infty)$ algebra. Since $\hat{\mathrm{gl}}(\infty)$ has a simpler structure, it is useful to carry out some explicit calculation by using this correspondence. As we show in this section, it is also useful to understand the structure of subalgebras in terms of $\hat{\mathrm{gl}}(\infty)$.

\footnotetext{
${ }^{1}$ We can also show that $\tilde{b}_{n+m}(w)$ divides $p(w-m) \tilde{b}_{n}(w-m) \tilde{b}_{m}(w)$.
} 
Let us start from the special case in which the differences of any roots of $\tilde{b}(w)=0$ and $p(w)=0$ are integers. We assume that $\tilde{b}(w)$ has the following form:

$$
\tilde{b}(w)=\prod_{i=1}^{N}\left(w-\lambda-k_{i}\right)^{\mu_{i}} \prod_{j=1}^{M}\left(w-\lambda-s_{j}\right)^{\nu_{j}}, \quad p(w)=\prod_{j=1}^{M}\left(w-\lambda-s_{j}\right)^{\rho_{j}},
$$

where $k_{i}, s_{j} \in \mathbb{Z}$ and $\mu_{i}, \nu_{j}, \rho_{j}$ are positive integers with $m \equiv \operatorname{Max}\left(\mu_{i}, \nu_{j}+\rho_{j}\right)$. We remark that general cases can also be handled by making their tensor products.

The $\mathrm{gl}(\infty)$ algebra we consider is defined by the commutation relation,

$$
\begin{aligned}
{\left[E^{(\mu)}(i, j), E^{(\nu)}(k, \ell)\right]=} & \theta(m-\mu-\nu \geq 0) \times \\
& \left(\delta_{j+k, 0} E^{(\mu+\nu)}(i, \ell)-\delta_{i+\ell, 0} E^{(\mu+\nu)}(k, j)\right. \\
& \left.+c^{(\mu+\nu)} \delta_{j+k, 0} \delta_{\ell+i, 0}(\theta(i \geq 0)-\theta(k \geq 0))\right),
\end{aligned}
$$

where $\mu$ and $\nu$ run from 0 to $m$. The quasifinite representation of $\hat{g l}(\infty)$ is defined by the highest weight state,

$$
E^{(\mu)}(i, j)|\lambda\rangle=0 \quad(i+j>0) \quad \text { and } \quad E^{(\mu)}(i,-i)|\lambda\rangle=q_{i}^{(\mu)}|\lambda\rangle .
$$

If we introduce $h_{i}^{(\mu)}=q_{i}^{(\mu)}-q_{i-1}^{(\mu)}+c^{(\mu)} \delta_{i, 0}$, the quasifiniteness of the module is achieved only when finite number of $h_{i}^{(\mu)}$ are non-vanishing [7].

The relation between the generators of $\hat{\mathrm{gl}}(\infty)$ and $W_{1+\infty}$ is given by,

$$
W\left(z^{r} f(D)\right)=\sum_{k \in \mathbb{Z}} \sum_{\mu=0}^{m} \frac{f^{(\mu)}(\lambda+k)}{\mu !} E^{(\mu)}(r+k,-k),
$$

for $r \neq 0$ [7]. For zero modes, we need to introduce c-number corrections [7, 10].

Since we are considering a subalgebra of $W_{1+\infty}$, we need to find a proper subalgebra of $\hat{\mathrm{gl}}(\infty)$ associated with it. Let us replace $f(D)$ in (19) by $\tilde{f}(D) p(D)$. We recognize that the coefficients of

$$
E^{(\mu)}\left(r,-s_{j}\right) \quad\left(r \in \mathbb{Z}, \quad \mu=0, \cdots, \rho_{j}-1\right),
$$

vanish on the right hand side of (19). This means that these generators will not appear in the image of the mapping (19). Thus, if we remove (20) from the algebra, we also have to remove the generators of the following form:[]

$$
E^{(\mu)}\left(s_{j}, r\right) \quad\left(r \in \mathbb{Z}, \quad \mu=m-\rho_{j}+1, \cdots, m\right) .
$$

${ }^{2}$ To prove this, we first remark that generators of the form, $E^{(\nu)}\left(*,-s_{j}\right)$ with $\nu=\rho_{j}, \cdots, m$, still remain in the algebra. The generators which has non-vanishing inner product with them are given in the form, $E^{(\nu)}\left(s_{j}, *\right)$ with $\nu=0, \cdots, m-\rho_{j}$. The other generators (21) have zero inner product with any states. 
The remaining generators will form a subalgebra of $\hat{\mathrm{gl}}(\infty)$, which will be called $\hat{\mathrm{gl}}(\infty)(\{s\})$ in the following.

The next step is to find a relation between the quasifinite representations of $W_{1+\infty}[p(D)]$ and $\hat{\mathrm{gl}}(\infty)(\{s\})$. This is again carried out by studying the relation (19) carefully. By putting $f(D)=\tilde{b}(D) p(D), r=-1$, it should become a null field when acting on the highest weight state. The only generators which may give nonvanishing states are, $E^{(\mu)}\left(-1+k_{i},-k_{i}\right)\left(\mu=0, \cdots, \mu_{i}-1\right)$ and $E^{(\mu)}\left(-1+s_{j},-s_{j}\right)\left(\mu=\rho_{j}, \cdots, \rho_{j}+\nu_{j}-1\right)$. Thus, applying the argument in [7], we can show that nonvanishing $h^{(\mu)}$ are given by $h_{k_{i}}^{(\mu)}\left(\mu=0, \cdots, \mu_{i}-1\right)$ and $h_{s_{j}}^{(\mu)}\left(\mu=\rho_{j}, \cdots, \rho_{j}+\nu_{j}-1\right)$. This information is enough for constructing a $\hat{\mathrm{gl}}(\infty)(\{s\})$ module from the highest weight state, which can be shown to be equivalent to the $W_{1+\infty}[p(D)]$ module we gave in section 3 .

\section{$5 \quad$ Free Field Realization of the $W_{\infty}$ Algebra}

In this section, we investigate the $W_{\infty}$ algebra $(p(D)=D)$ as an example.

We first consider the free-field realization of $W_{\infty}$, introducing complex bosons, $\bar{\varphi}(z)=$ $\bar{q}+\bar{\alpha}_{0} \log z-\sum_{n \neq 0} \frac{1}{n} \bar{\alpha}_{n} z^{-n}, \varphi(z)=q+\alpha_{0} \log z-\sum_{n \neq 0} \frac{1}{n} \alpha_{n} z^{-n}$ with commutation relations: $\left[\bar{\alpha}_{n}, \alpha_{m}\right]=n \delta_{n+m, 0},\left[\bar{\alpha}_{0}, q\right]=1,\left[\alpha_{0}, \bar{q}\right]=1$, i.e., with the propagator $\bar{\varphi}(z) \varphi(w) \sim$ $\log (z-w)$. The $W_{\infty}$ algebra with $C=-1\left(\widetilde{c}_{V i r}=2\right)$ is then realized in the following way [5]:

$$
\widetilde{W}\left(z^{n} f(D)\right)=-\oint \frac{d z}{2 \pi i}: \partial \bar{\varphi}(z) z^{n+1} f(D+1) \partial \varphi(z):=-\sum_{m \in \mathbb{Z}} f(-m): \bar{\alpha}_{n-m} \alpha_{m}: .
$$

We remark that

$$
\left[\widetilde{W}\left(z^{n} f(D)\right), \bar{\alpha}_{m}\right]=m f(m) \bar{\alpha}_{n+m}, \quad\left[\widetilde{W}\left(z^{n} f(D)\right), \alpha_{m}\right]=m f(-n-m) \alpha_{n+m} .
$$

There are two kinds of highest weight states for such realization [5, 6]:

$$
\begin{aligned}
|p, \bar{p}\rangle & =: \exp (p \bar{\varphi}(0)+\bar{p} \varphi(0)):|0\rangle \\
|N\rangle & = \begin{cases}\left(\alpha_{-1}\right)^{N}|0\rangle & (N \geq 1) \\
|0\rangle & (N=0) \\
\left(\bar{\alpha}_{-1}\right)^{-N}|0\rangle & (N \leq-1)\end{cases}
\end{aligned}
$$

where $p, \bar{p} \in \mathbb{C}, N \in \mathbb{Z}$ and $|0\rangle$ is defined by $\bar{\alpha}_{n}|0\rangle=\alpha_{n}|0\rangle=0$ ( $\left.n \geq 0\right)$. Using eq. (23), we can show that the weight $\widetilde{\Delta}(x)$ and the characteristic polynomials $\tilde{b}_{n}(w)$ are given by the following: for $|p, \bar{p}\rangle$

$$
\begin{aligned}
\widetilde{\Delta}(x) & =p \bar{p} \\
\tilde{b}_{n}(w) & =\left\{\begin{array}{lll}
{[w-1]_{n-1}} & \text { if } & p \bar{p}=0 \\
{[w]_{n+1}} & \text { if } & p \bar{p} \neq 0
\end{array}\right.
\end{aligned}
$$


and for $|N\rangle$

$$
\begin{aligned}
\widetilde{\Delta}(x) & =|N| e^{\operatorname{sgn}(N) x}, \\
\tilde{b}_{n}(w) & =\left\{\begin{array}{lll}
{[w-1]_{n-1} \cdot(w-n-1)} & \text { if } \quad N \geq 1 \\
{[w-1]_{n-1}} & \text { if } \quad N=0 \\
{[w-1]_{n-1} \cdot(w+1)} & \text { if } \quad N \leq-1,
\end{array}\right.
\end{aligned}
$$

We can illustrate our general theory given in the previous section from this example. For $|p, \bar{p}\rangle$, we can take $\Delta(x)=p \bar{p} x$. Then $f_{n}(w)$ is equal to $b_{n}(w)=[w]_{n}$ for $p \bar{p}=0$. Thus $\tilde{b}_{n}(w)$ are given by eq. (15). But for $p \bar{p} \neq 0, f_{n}(w)=[w]_{n+1} w(w-n)$ does not agree with $b_{n}(w)=\left([w]_{n+1}\right)^{2}$. So the polynomials in eq. (15) may not be of minimal degree, and actually they are not.

Using eq. (23) and a formula in [6, 13], we can write down the full character of the representation $|p, \bar{p}\rangle$ with $p \bar{p} \neq 0$ as

$$
\operatorname{ch}_{p, \bar{p}}=\operatorname{tr} e^{\sum_{k=0}^{\infty} g_{k} \widetilde{W}\left(D^{k}\right)}=e^{-p \bar{p} g_{0}} \prod_{n=1}^{\infty}\left(1-e^{\sum_{k=0}^{\infty} g_{k} n^{k+1}}\right)^{-1}\left(1-e^{-\sum_{k=0}^{\infty} g_{k}(-n)^{k+1}}\right)^{-1},
$$

and the generating function of the full characters of the representations $|N\rangle, \operatorname{ch}_{N}=$ $\operatorname{tr} e^{\sum_{k=0}^{\infty} g_{k} \widetilde{W}\left(D^{k}\right)}=e^{-\sum_{k=0}^{\infty}|N|(\operatorname{sgn}(N))^{k} g_{k}} \mathrm{ch}_{N}^{\prime}$, as

$$
\sum_{N \in \mathbb{Z}} t^{N} \operatorname{ch}_{N}^{\prime}=\prod_{n=1}^{\infty}\left(1-t^{-1} e^{\sum_{k=0}^{\infty} g_{k} n^{k+1}}\right)^{-1}\left(1-t e^{-\sum_{k=0}^{\infty} g_{k}(-n)^{k+1}}\right)^{-1} .
$$

We here make a comment. The $W_{1+\infty}$ algebra is known to be realized by free fermions or bc ghosts [14, 8]. By restricting quasifinite representations of $W_{1+\infty}$, we obtain those of $W_{\infty}$ or more generally $W_{1+\infty}[p(D)]$.

\section{Discussion}

We have constructed a series of subalgebras of $W_{1+\infty}$ parametrized by polynomials $p(w)$, in which $W_{\infty}$ corresponds to $p(w)=w$, and studied their quasifinite representations.

Although $W_{\infty}$ is a subalgebra of $W_{1+\infty}$, its representation theory is nontrivial. There exist more null states than the $W_{1+\infty}$ case. Although full character formulae of quasifinite representations of $W_{1+\infty}$ were recently obtained [10, 11], it is difficult to derive the (full) character formulae of $W_{\infty}$ directly from those of $W_{1+\infty}$. We would like to report on this issue in our future communication.

Acknowledgments: S.O. would like to thank members of YITP for their hospitality. This work is supported in part by Grant-in-Aid for Scientific Research from Ministry of Science and Culture. 


\section{Appendix: Kac Determinant at Lower Degrees}

In this appendix, we give the Kac determinant for the $p(w)=w$ and $\tilde{b}(w)=w-\lambda$ case. In this case, the generating function for the highest weight can be written as $\Delta(x)=C_{1}\left(e^{\lambda x}-1\right) /\left(e^{x}-1\right)$ with $C_{1}$ some complex number.

For the first three levels, the relevant ket states are,

Level $1 \quad \widetilde{W}\left(z^{-1}\right)|\lambda\rangle$,

Level $2 \quad \widetilde{W}\left(z^{-2}\right)|\lambda\rangle, \widetilde{W}\left(z^{-2} D\right)|\lambda\rangle, \widetilde{W}\left(z^{-2} D^{2}\right)|\lambda\rangle, \widetilde{W}\left(z^{-1}\right)^{2}|\lambda\rangle$,

Level $3 \widetilde{W}\left(z^{-3}\right)|\lambda\rangle, \widetilde{W}\left(z^{-3} D\right)|\lambda\rangle, \widetilde{W}\left(z^{-3} D^{2}\right)|\lambda\rangle, \widetilde{W}\left(z^{-3} D^{3}\right)|\lambda\rangle, \widetilde{W}\left(z^{-3} D^{4}\right)|\lambda\rangle$,

$$
\begin{aligned}
& \widetilde{W}\left(z^{-1}\right) \widetilde{W}\left(z^{-2}\right)|\lambda\rangle, \widetilde{W}\left(z^{-1}\right) \widetilde{W}\left(z^{-2} D\right)|\lambda\rangle, \\
& \widetilde{W}\left(z^{-1}\right) \widetilde{W}\left(z^{-2} D^{2}\right)|\lambda\rangle, \widetilde{W}\left(z^{-1}\right)^{3}|\lambda\rangle .
\end{aligned}
$$

Corresponding bra states may be given by changing $z^{-r}$ into $z^{r}$.

We compute the Kac determinant up to level 4, and the result is

$$
\begin{aligned}
\operatorname{det}[1] & \propto C_{1} \lambda(\lambda-1), \\
\operatorname{det}[2] & \propto C_{1}^{3}\left(C_{1}+1\right) C_{2}(\lambda+1) \lambda^{5}(\lambda-1)^{5}(\lambda-2), \\
\operatorname{det}[3] & \propto C_{1}^{7}\left(C_{1}+1\right)^{3}\left(C_{1}-2\right) C_{2}^{3}(\lambda+2)(\lambda+1)^{4} \lambda^{14}(\lambda-1)^{14}(\lambda-2)^{4}(\lambda-3), \\
\operatorname{det}[4] & \propto\left(C_{1}+1\right) C_{1}^{18}\left(C_{1}-1\right)^{9}\left(C_{1}-2\right)^{3}\left(C_{1}-3\right) C_{2}^{9}\left(C_{2}-1\right) \\
& \times(\lambda+3)(\lambda+2)^{4}(\lambda+1)^{13} \lambda^{42}(\lambda-1)^{42}(\lambda-2)^{13}(\lambda-3)^{4}(\lambda-4),
\end{aligned}
$$

where $C_{2} \equiv C-C_{1}$.

\section{References}

[1] I. Bakas, Phys. Lett. B228 (1989) 57-63.

[2] C.N. Pope, L.J. Romans and X. Shen, Phys. Lett. B236 (1989) 173-178; Nucl. Phys. 339B (1990) 191-221.

[3] C.N. Pope, L.J. Romans and X. Shen, Phys. Lett. B242 (1990) 401-406.

[4] C.N. Pope, L.J. Romans and X. Shen, Phys. Lett. B245 (1990) 72-78.

[5] I. Bakas and E. Kiritsis, Nucl. Phys. B343 (1990) 185-204, Erratum B350 (1991) 512; Mod. Phys. Lett. A5 (1990) 2039-2050; Prog. Theor. Phys. Suppl. No.102 (1990) 15-38.

[6] S. Odake, Int. J. of Mod. Phys. A7 (1992) 6339-6355. 
[7] V. Kac and A. Radul, Comm. Math. Phys. 157 (1993) 429-457.

[8] Y. Matsuo, Phys. Lett. B326 (1994) 95.

[9] H. Awata, M. Fukuma, Y. Matsuo and S. Odake, Determinant Formulae of QuasiFinite Representation of $W_{1+\infty}$ Algebra at Lower Levels, preprint YITP/K-1054, UT669, SULDP-1994-1, hep-th/9402001, (January 1994), to be published in Phys. Lett. B.

[10] H. Awata, M. Fukuma, Y. Matsuo and S. Odake, Character and Determinant Formulae of Quasifinite Representation of the $W_{1+\infty}$ Algebra, preprint YITP/K-1060, YITP/U-94-17, SULDP-1994-3, hep-th/9405093, (May 1994).

[11] E. Frenkel, V. Kac, A. Radul and W. Wang, $\mathcal{W}_{1+\text { inf }}$ and $\mathcal{W}\left(g l_{N}\right)$ with central charge $N$, preprint, hep-th/9405121, (May 1994).

[12] H. Awata, M. Fukuma, Y. Matsuo and S. Odake, Quasifinite Highest Weight Modules over the Super $\mathcal{W}_{1+\infty}$ Algebra, preprint YITP/K-1055, UT-670, SULDP-1994-2, hepth/9404041, (March 1994).

[13] H. Awata, M. Fukuma, S. Odake and Y. Quano, Eigensystem and Full Character Formula of the $\mathcal{W}_{1+\infty}$ Algebra with $c=1$, preprint YITP/K-1049, SULDP-1993-1, RIMS-959, hep-th/9312208 (December 1993), to be published in Lett. Math. Phys.

[14] E. Bergshoeff, C.N. Pope, L.J. Romans, E. Sezgin and X. Shen, Phys. Lett. B245 (1990) 447-452. 